\title{
Simulation Case Study: Using Simscape for Human Knee Joint Models
}

\author{
Ruth Leskovar ${ }^{*}$, Andreas Körner, Felix Breitenecker \\ ${ }^{1}$ Institute of Analysis and Scientific Computing, TU Wien, Wiedner Hauptstraße 8-10, 1040 Vienna, Austria; \\ *ruth.leskovar@tuwien.ac.at
}

SNE 29(2), 2019, 101-104, DOI: 10.11128/sne.29.sn.10477 Received: March 30, 2019 (Selected ASIM GMMS/STS 2019 Conference Publication); Accepted; May 10, 2019

SNE - Simulation Notes Europe, ARGESIM Publisher Vienna, ISSN Print 2305-9974, Online 2306-0271, www.sne-journal.org

Abstract. This contribution presents a knee model implemented in Simscape ${ }^{\mathrm{TM}}$ and analyses its usability regarding biomechanical aspects. The model simulates the flexion of a human knee. It contains the three bones of the human knee, which are linked together by two revolute joints and one spring damper element representing the patellar tendon. This illustrates a simplification of the human knee joint due to the restriction of degrees of freedom. Finally, this work discusses the advantages and disadvantages of using the multibody library in Simscape for biomechanical models.

\section{Introduction}

In the research field of biomechanics, mathematical models for anatomic joints play an important role analysing kinematics and kinetics in the human body. Mainly, two different modelling approaches are used, models based on partial differential equations and multibody systems. They differ in their mathematical description and therefore in application fields as well.

Models described by partial differential equations (PDEs) depend on time and space, which gives the opportunity to analyse even small deformations, which take place in human bone and soft tissue, as ligaments and tendons, under repeated loads.

Multibody models are described by ordinary differential equations (ODEs), which leads to final solutions dependent on time only. These models do not require a precise description as PDE models, because detailed information about the geometries of the bodies does not influence the solution of the model strongly. Multibody models are often used for investigating gross motion and interaction of various connected bodies. Here, a de- scription of movement change over time is needed and no deformations in tissues are investigated.

One possible application is the gait analysis. The study of motion sequences, in case of injuries compared to a normal gait cycle, can give insights to rehabilitation and therapy techniques. Further on, biomechanical models, which simulate the human walk, can be extended to various walking scenarios, as running or climbing stairs.

Results gained from these models can be used in the development of prostheses to improve functions of leg prostheses. For example, multibody models are developed for the investigation of interactions taking place in the human body of amputees wearing prostheses during various falling scenarios as it is done in [1].

\section{Multibody Modelling}

Physical modelling describes a system based on fundamental physical laws. This technique is often used for systems, where no mathematical equations describing the dynamical behaviour of the system are known. Multibody models are based on the physical modelling approach and describe relative motion between different bodies and the resulting dynamics. Multibody systems consist of bodies and joints, which link them together. Bodies are defined by their physical properties, as mass, centre of mass, density, inertial rotation, etc. There exist various types of joints, which differ in their amount and properties of degrees of freedom, resulting in rotational or translational movement respectively.

Figure 1 shows two rigid bodies, which are connected by a kinematic joint. The positions of the bodies, defined by their local coordinates, can be described in respect to the global coordinate system as it is explained more detailed in [2]. Since the physical model building process does not require mathematical equations, the development of multibody models is often simpler and faster than other methods. The description of a system by PDEs requires more detailed information about 


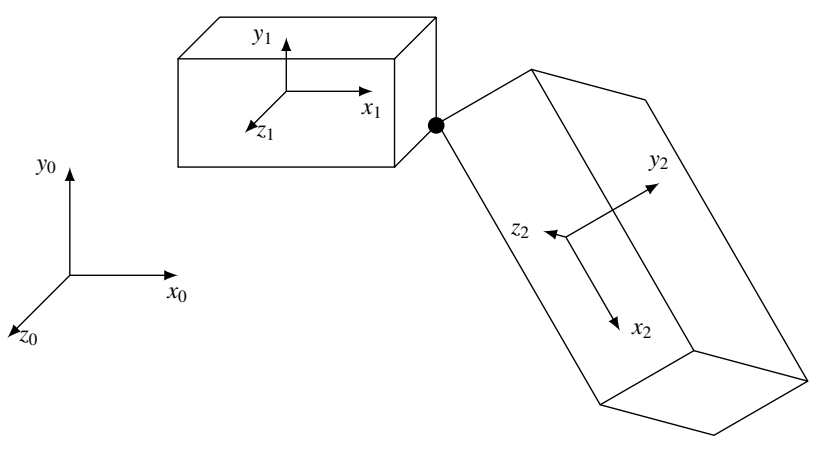

Figure 1: Illustration of a multibody system with local coordinates, indicated by $x_{1}$ and $x_{2}$, and the global coordinate system, indicated by $x_{0}$.

geometries defining the included bodies and the numerical solving procedure is more complex than using ODE solver, which are sufficient for multibody models. Furthermore, the physical modelling approach can be used to detect mathematical equations of complex systems to describe their dynamical behaviour. The combination of components, whose dynamics are already known, leads to the desired system description. The analysis and further investigation of simulation results gives the possibility to describe the dynamics by mathematical equations.

In multibody models, the relative motion of a rigid body, which results by applying a force $\boldsymbol{F}$, is given by the set of a second order ordinary differential equations

$$
M \ddot{\boldsymbol{x}}+J_{\boldsymbol{x}}^{T} \lambda=\boldsymbol{F},
$$

with the mass matrix $M$ of the system, the coordinates $\boldsymbol{x}$ of the investigated body, the corresponding Jacobian matrix $J$ and the Lagrange multipliers $\lambda$. The derivation can be conducted by using the Lagrange formalism and is explained in [3].

As already mentioned above, multibody models are used to analyse kinematics between bodies in biomechanics. For example, they are used to analyse the interactions in the human body, which take place during motion.

\section{Conceptual Model for the Human Knee}

The platform https: / / simtk . org offers a repository of biomechanical models. Researchers can share their work, including simulation models and corre- sponding files as geometries or data. The open access strategy facilitates the development of new models.

The following model simulates the flexion of a human knee and is based on the work of [4], [5] and [6]. The investigated models are mainly implemented in Adams ${ }^{\mathrm{TM}}$, a multibody dynamics simulation software. These models describe the flexion of the tibia and the involved movement of the patella in respect to a fixed femur after applying a force at the tibia. Similar to the anatomy of the human knee, ligament forces are included, connecting the bones together and predetermining the direction of motion. Additionally, contact forces influence the movement to prevent interpenetration of the bones.

The usage of the multibody library for Simscape, embedded in the Simulink ${ }^{\circledR}$ environment, requires the introduction of joints, otherwise no movement is possible. A possible simplification of the knee joint reduces the degrees of freedom in the knee to one rotational as it is stated in [7]. For the analysis of load distribution in the knee joint, this simplification is sufficient. This rotational degree of freedom describes the flexion of the tibia and is represented by one revolute joint. This revolute joint $j_{1}$, connecting femur and tibia, is placed in the last third of the femoral condyle as it is depicted in Figure 2. A second revolute joint $j_{2}$ is introduced, linking the patella to the femur and describing the movement of the patella. This joint is situated at the centre of mass of the femur to ensure that the patella is sliding between the femoral condyles at the front side. In the anatomy of the human knee, the patella is situated in the patellar tendon, which connects the quadriceps to the tibia. In biomechanics, tendons are often implemented using
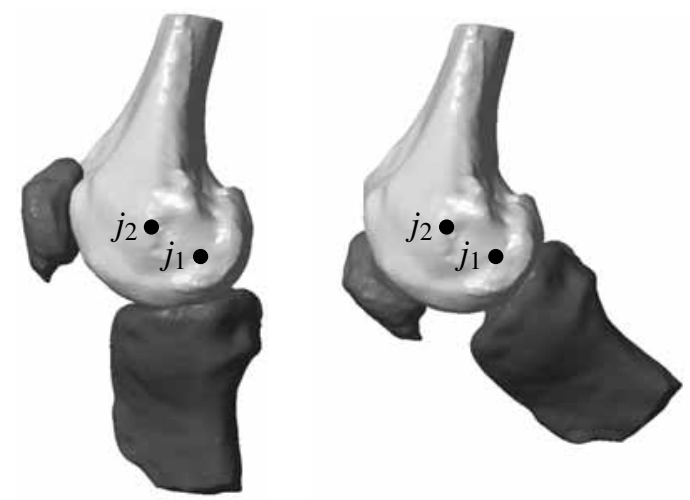

Figure 2: Visualisation of the joint centres in the knee model in the initial position left and in flexion right. 
spring damper forces. Therefore, one spring damper element is introduced, which connects tibia and patella. This component applies a linear force acting reciprocally between the connected bodies. This force $f$ is proportional to the distance $x$ between the connected bodies and the resulting velocity $\dot{x}$. It is calculated by

$$
f=k \cdot(x-l)+D \cdot \dot{x},
$$

with the spring constant $k$ and damper coefficient $D$. The natural length of the spring is given by $l$. This spring damper force does not imply any movement to the bodies, but ensures that the patella is sliding following the rotation of the tibia. The tibia starts to move only after an applied torque at the joint $j_{1}$.

\section{Simulation Model in Simscape for the Human Knee}

Simscape is embedded in the Simulink environment and is intended for the development of physical systems. The library comprises elements for driveline, electrical, fluids and multibody models. Due to the embedment to Simulink, it is possible to use components of Simulink as well. For that purpose, special blocks convert the different signal types from 3D of Simscape to 1D of Simulink and vice versa.

The construction of a simulation model in Simscape is similar to Simulink and realised by drag and drop of components. The final model structure for the flexion of a human knee is shown in Figure 3. Each block represents one component of the model. The global parameters, as gravity and solver settings as well as the global reference frame, are defined in blocks too. Without these blocks, a simulation run of a Simscape model is not possible.

The knee model consists of three bones, femur, tibia and patella, which are implemented as rigid bodies. The corresponding blocks contain stl-files defining their geometry and shape as well as physical parameters, namely mass, centre of mass and inertial rotation. These files and parameters are given with the Adams models of [4], [5] and [6] and represent data of the right knee of a 77 year old man.

The stiffness of the spring and damping coefficient for the patellar tendon between tibia and patella are the same values as in the Adams model. The rigid transforms between the components assure the correct attachment points of the tendon to the bones and the right position of joint centres, respectively.
The revolute joints contain parameters for spring stiffness and damping coefficient of the joint and are summarised in Table 1. These parameters are not the same as in the Adams models, because there no rotational joints are considered. These models deal with ligaments only, which contain parameters for translational movements. The conversion of these parameters for rotational movement requires knowledge about rotational stiffness and is not accessible in this case.

\begin{tabular}{|c|cc|}
\cline { 2 - 3 } \multicolumn{1}{c|}{} & Spring stiffness & Damping coefficient \\
\hline$j_{1}$ & $553.5 \frac{\mathrm{N} \mathrm{mm}}{\mathrm{deg}}$ & $1 \frac{\mathrm{N} \mathrm{mm} \mathrm{s}}{\mathrm{deg}}$ \\
$j_{2}$ & $33 \frac{\mathrm{N} \mathrm{mm}}{\mathrm{deg}}$ & $1 \frac{\mathrm{N} \mathrm{mm} \mathrm{s}}{\operatorname{deg}}$ \\
\hline
\end{tabular}

Table 1: Parameters for the joints.

Therefore, the parameters for the joints are calculated by calibration and comparing the outputs from the Adams and Simscape model. Both model do not show the same behaviour due to the already discussed restrictions. One more subsequent difference from the model description is the input. In the Adams model, a force is acting at the tibia in posterior direction. The Simscape model requires a torque $\tau$ acting on the joint $j_{1}$. The torque $\tau$ is calculated by using geometrical basics and is finally given by

$$
\tau(t)=28.8 \cdot \sin (2 \pi \cdot 0.125(t-1)) \cdot \mathrm{H}(t-1) \mathrm{Nm},
$$

with the step function $\mathrm{H}$. The acting torque is implemented in Simscape by using Simulink blocks.

The output of the simulation model is the resulting angle between femur and tibia representing the flexion and extension of the human knee. Figure 4 shows the angle of the Adams model and the Simscape model. The comparison of the output leads to the missing joint parameters. The non-linearity of the Adams model, coming from the ligaments, results in a more steep waveform than the sine wave from the Simscape model.

\section{Conclusion and Outlook}

The presented knee model is a simplification of the human knee joint and it shows the possibilities and restrictions of using the multibody library in Simscape for biomechanical models. The usage of Simscape for building multibody models is a good option due to the embedment in the Simulink environment. This allows 


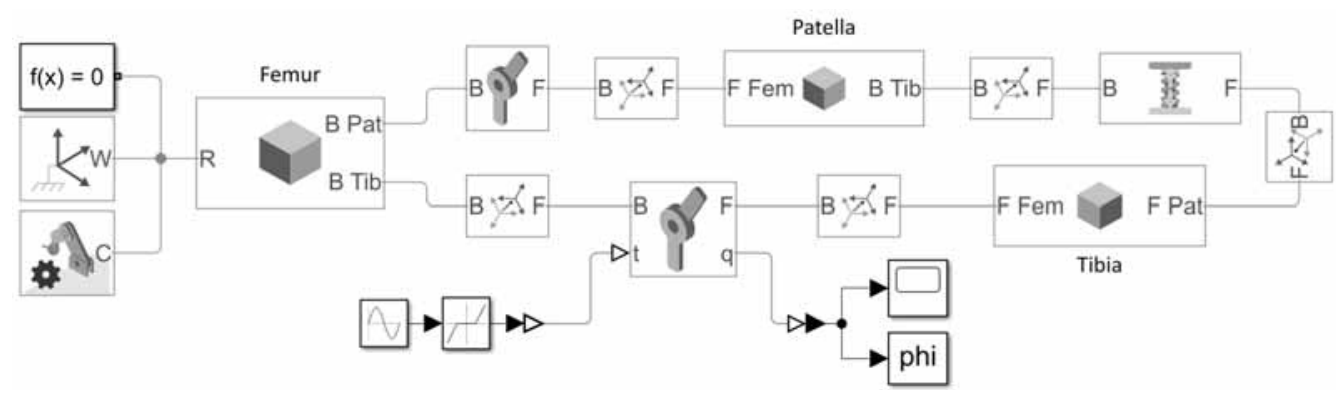

Figure 3: Block structure of the multibody model for the human knee implemented in Simscape.

to use block elements from other libraries and all tools which are available in Simulink. Moreover, the postprocessing in MATLAB ${ }^{\circledR}$ offers many possibilities. This gives flexibility in the development and extension of the model and later in the analysis. For example, the construction of more complex model structures and combination with system based modelling approaches allows to build feedback loops, which extend the areas of application for biomechanical models.

Since MathWorks ${ }^{\circledR}$ does not focus on physical modelling, the library of Simscape is restricted regarding some aspects. Anatomic joints are complex structures due to their composition of various tissues as bones, ligaments and tendons. In order to build a model which fulfils the biomechanical properties of the human knee joint, the consideration of a revolute joint is insufficient. The incorporation of crucial and collateral ligaments to the model increases the degrees of freedom and improves a realistic movement. As it is discussed in [8], ligaments show a non-linear behaviour regard-

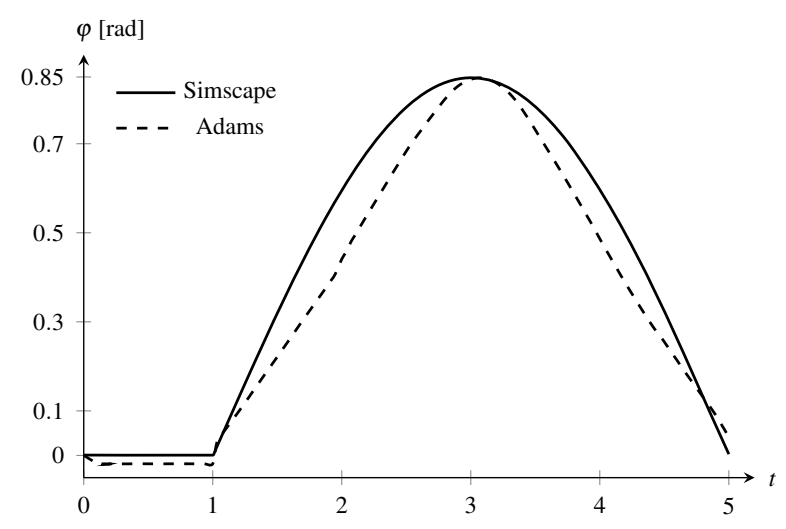

Figure 4: Angle $\varphi$ between femur and tibia during flexion of the knee for the Adams and Simscape model. ing their stress-strain relationship. Therefore, they can not be modelled with linear spring damper elements but using external functions. Nevertheless, the Simscape multibody library requires the use of joints to create the preconditions for the movements.

\section{References}

[1] Welke B, Schwarze M, Hurschler C, Calliess T, Seehaus F. Multi-body simulation of various falling scenarios for determining resulting loads at the prosthesis interface of transfemoral amputees with osseointegrated fixation. Journal of Orthopaedic Research. 2013; 31(7):1123-1129.

[2] Georg Rill TS. Grundlagen und Methoden der Mehrkörpersimulation, vol. 3. Springer Vieweg. 2017.

[3] Quental C, Folgado J, Ambrósio J, Monteiro J. A multibody biomechanical model of the upper limb including the shoulder girdle. Multibody System Dynamics. 2012;28(1-2):83-108.

[4] Guess TM, Thiagarajan G, Kia M, Mishra M. A subject specific multibody model of the knee with menisci. Medical Engineering and Physics. 2010;32(5):505-515.

[5] Guess TM, Liu H, Bhashyam S, Thiagarajan G. A multibody knee model with discrete cartilage prediction of tibio-femoral contact mechanics. Computer Methods in Biomechanics and Biomedical Engineering. 2013; 16(3):256-270.

[6] Bloemker KH, Guess TM, Maletsky L, Dodd K. Computational Knee Ligament Modeling Using Experimentally Determined Zero-Load Lengths. The Open Biomedical Engineering Journal. 2012;6:33-41.

[7] Brinckmann P, Frobin W, Leivseth G, Drerup B. Orthopädische Biomechanik. MV Wissenschaft. 2012.

[8] Blankevoort L, Huiskes R. Ligament-Bone Interaction in a Three-Dimensional Model of the Knee. Journal of Biomechanical Engineering. 1991;113(3):263. 